\title{
Cervical cancer is not just a young woman's disease
}

Susan Sherman and colleagues argue that the upper age limit for cervical screening needs revisiting and call for awareness campaigns to target older as well as younger women

\section{Susan M Sherman senior lecturer in psychology ${ }^{1}$, Alejandra Castanon epidemiologist ${ }^{2}$, Esther Moss consultant gynaecological oncologist ${ }^{3}$, Charles W E Redman consultant gynaecological oncologist $^{4}$}

${ }^{1}$ School of Psychology, Keele University, Keele, Staffs ST5 5BG, UK; ${ }^{2}$ Wolfson Institute of Preventive Medicine, Barts and The London School of Medicine and Dentistry, London, UK ; ${ }^{3}$ Department of Gynaecological Oncology, University Hospitals of Leicester NHS Trust, Leicester, UK ; ${ }^{4}$ Department of Obstetrics and Gynaecology, Royal Stoke University Hospital, Stoke on Trent, UK

Cervical screening programmes in many countries stop at around the age of 65 and much of the focus is often on younger women. For example, recent media campaigns in England and Wales have centred on lowering the age at first screening. Comparatively little attention has been given to older women despite the fact that they account for about a fifth of cases each year and half of deaths. ${ }^{12}$ Of the 3121 women diagnosed on average each year between 2009 and 2011 in the UK, only 64 were younger than 25 compared with 616 who were older than 65. ${ }^{1}$ As the population ages, this number of older women affected is set to increase. We argue that screening programmes should reflect this.

\section{Age distribution of cases}

The raw statistics conceal the full impact of cervical cancer on older women. Although the absolute figures decrease with increasing age, when the rate of new cases per 100000 women is considered, a second peak in diagnoses after the age of 65 becomes apparent (table $\downarrow$ ). The case rate gradually decreases from 19.7 new diagnoses per 100000 women aged 30-34 to a low of 8.6/100 000 women aged $65-69$, but then rises again, reaching 12.5/100 000 in women aged 80-84. Furthermore, mortality data for the UK from 2010-12 show only seven deaths a year from cervical cancer in women younger than 25 but 449 deaths in women older than 65-nearly half of the total average deaths from cervical cancer in any year. ${ }^{2}$

Although recent research by Castanon and colleagues suggests that women aged 65 who have had regular screening with negative results have a relatively low 20 year risk of $8 / 10000$ women, it also reports that for those women who have not been screened between the ages of 50 and 64, the 20 year risk rises to $49 / 10000$ women. ${ }^{3}$ This raises several issues.

\section{Screening uptake falls in older women}

The proportion of women in England in 2013 who had been screened in the past five years dropped with age, from $82.4 \%$ of 50-54 year olds to $75.9 \%$ of $55-59$ year olds and $72.7 \%$ of 60-64 year olds. ${ }^{4}$ These numbers are set to fall further because uptake is decreasing across all age groups. More than 25\% of older women would therefore not be in the low risk group defined by Castanon and colleagues. ${ }^{3}$

The fall in uptake suggests that as women age they perceive cervical screening to be less important. This is supported by Waller and colleagues, ${ }^{5}$ who found that only $12 \%$ of $55-64$ year old women cited not attending screening as a risk factor for cervical cancer. In addition, only $2.1 \%$ of participants across their whole sample identified older age as a risk factor, suggesting that it is perceived as being a younger woman's disease. This interpretation is strengthened by the fact that the age related decline in screening uptake seems to be specific to cervical screening. The 2010-11 breast cancer screening data show that $70.7 \%$ of invited women aged $45-49$ attended breast screening, increasing to $74.9 \%$ in women aged $60-64{ }^{6}$

The perception of cervical cancer as a younger woman's disease may have been reinforced by the high profile death in March 2009 of television celebrity Jade Goody at the age of 27. Although an extra half a million women were screened between 2008 and 2009, the increase was greatest in the under $50 \mathrm{~s},{ }^{7}$ with women aged 26-35 years more likely to be influenced by the story than those aged $56-64$ years. ${ }^{8}$ The perception of cervical cancer as a young woman's problem is likely to have been further enhanced by high profile media campaigns to reduce the age of screening after the death of a few young women as well as the introduction of the human papillomavirus (HPV) vaccination for teenage girls in 2008. Furthermore, the fact that 
the screening programme ends at 65 implicitly suggests that older women are no longer at risk.

\section{Older women present with later stages of cancer}

As well as having high rates of cervical cancer, women over 65 are more likely to die from it than younger women. ${ }^{2}$ Increased age at the time of diagnosis may contribute to higher mortality, but lack of awareness that age increases risk may mean women do not present until their cancer is at an advanced stage. This could be exacerbated by a lack of knowledge of the symptoms of cervical cancer, although there has been little research into awareness in this age group. Low et al explored awareness of cervical cancer risk factors and symptoms in British women aged 16-94 divided into four age categories (16-24, 25-39, $40-59, \geq 60$ ). Older age predicted increased risk factor and symptom recognition, but overall awareness was low. ${ }^{9}$

Even if they know the relevant symptoms, age related changes may mean older women do not detect them at an early stage. If sexual activity is reduced through declining libido or loss of a partner, symptoms such as pain or discomfort during intercourse or bleeding afterwards may not be identified. Reduced olfactory sensitivity ${ }^{10}$ may also delay the identification of unpleasant smelling vaginal discharge.

Examination of data on age and stage of presentation from 2753 women presenting during 1993-7 shows that only $2 \%$ of women aged $\geq 70$ presented with stage $1 \mathrm{~A}$ compared with $20 \%$ of women aged 40-54. ${ }^{11}$ More recent data support a later stage at diagnosis for older women. In 2009-10, 3.6\% of women $\geq 65$ had a diagnosis of stage $1 \mathrm{~A}, 19.9 \%$ stage $1 \mathrm{~B}$, and $51.1 \%$ stage 2 or higher. For women under the age of 65 , these figures were $39.2 \%, 30.5 \%$, and $19.4 \%$ respectively. ${ }^{12}$

\section{Upper age limit for screening}

Although the definition of low risk can be debated, 65 is indisputably an arbitrary cut-off point for screening set in 1988 when life expectancy at birth for a woman in the UK was nearly five years lower than today: 78.2 compared with 82.7 in $2013 .{ }^{13}$ It is true that problems obtaining an adequate and accurate cervical cytological sample increase with age. These include difficulties with the examination itself, such as pain caused by musculoskeletal disorders or vaginal atrophy. Vaginal atrophy can also lead to increased false positive results, while problems accessing the transformation zone, which is located higher in the endocervical canal in older women, can lead to higher false negative results. ${ }^{14}$ However, these conditions vary from woman to woman, and for many healthy older women, 65 may be too young for any attendant discomfort or risks to outweigh the benefit of additional screening. Furthermore, these problems have mainly been identified for cytology and we are now at a crossroads in cervical screening whereby HPV testing is likely to become the primary screening tool and the same problems may not be relevant.

Age in itself is not a reason for stopping screening. Routine breast cancer screening in the UK continues until 70 (rising to 73 from 2016 in England) and screening is available on demand every three years after 70 . The upper age limit for routine bowel screening in England is currently being extended from 69 to 74 (in line with Scotland) and thereafter on demand. Ending cervical screening at an age at which only half of the women who will die from the disease have done so seems inappropriate. Furthermore, using the justification of a low risk, which pertains only to women who have been regularly screened, disadvantages the $25 \%$ or more of women who have not been; these women are likely to be non-white and to come from lower educational backgrounds. ${ }^{15}$

\section{Case for screening older women and some possible solutions}

Elit's literature review exploring cervical screening in older women reported that screening is beneficial until at least age 69 in terms of preventing occurrence of and death from cervical cancer. ${ }^{14}$ With the exception of the Castanon et al study, ${ }^{3}$ the other studies reviewed found that the protective effect of screening in older women was up to around five years. While acknowledging the difficulties and costs of screening older women, she suggests that "the stopping age of screening perhaps should depend in part on the woman's characteristics and preferences."

Since female life expectancy has increased by almost five years since the screening programme began in the UK, we should consider raising the end age to 70 years. In addition, or alternatively, cervical screening could be provided on demand every five years for women over 65 (or 70), as is the case for breast and bowel screening, possibly with an HPV test on exit from the screening programme. Previous research in the $\mathrm{UK}^{16}$ has explored how older adults (60-74 years) feel about continuing breast and bowel screening; $78 \%$ of respondents did not agree with age based stoppage policies. However, although $83 \%$ wanted a strong recommendation to opt in after the cut-off point, only $27 \%$ said they would be "very likely" to request screening, with another $33 \%$ saying they would be "quite likely" to do so. This suggests that there would be public support for increasing the availability of screening, while providing some reassurance to budget holders that not all eligible women would take the opportunity.

Increasing the age limit does not address the fact that eligible women need to attend screening to benefit. Since there is already a decline in uptake of cervical screening in women over the age of 55, efforts must be made to increase cervical screening uptake in this group. If older women believe that they are not at risk or are at reduced risk of cervical cancer because of their age and there is no ongoing health literacy aimed at those women, there is no reason for them to attend routine screening, look out for symptoms, or re-enter the screening programme if they have previously opted out. Awareness could be increased for relatively little cost by giving older women information leaflets when they attend primary care for other interventions such as a flu jab or when they have breast cancer screening.

An alternative approach is to send older women a self sampling kit for HPV. Most studies of self sampling in women do not include women beyond the age of 65 . However, a study in Canada comparing self sampling with physician cervical sampling for HPV found $47.9 \%$ of older women (age 50-80) preferred self sampling or had no preference. ${ }^{17}$ A recent study found that an in-home return mail kit sent to women aged 30-65 who had not previously responded to a call to screening was significantly more effective than a recall letter in eliciting a response. ${ }^{18}$ Uptake of follow-up cervical screening among the women with a positive HPV result was $91 \%$. This suggests that posting kits to older women could reduce the risk for those who have not taken full advantage of the call-recall system or at least enable any cancer to be diagnosed at an earlier stage with the potential to increase healthy years of life expectancy. With the numbers of older women developing cervical cancer likely to rise as longevity increases, urgent action is needed. 


\section{Summary points}

Women over 65 account for a fifth of new cases of cervical cancer and half of deaths in the UK

Attendance for routine screening falls with age

Health campaigns are needed to increase awareness of risk among older women

The upper age limit of the cervical screening programme needs revisiting

Contributors and sources: SS conducts research exploring awareness of risk factors for cervical cancer and HPV in the general population as well as research with women who have cervical cancer and clinicians. AC is an epidemiologist whose research focuses on the evaluation of screening programmes, in particular cervical screening. EM and CWER are consultant gynaecological oncologists. All authors assisted with drafting the article and all have approved the final version. SS is the guarantor.

Competing interests: We have read and understood BMJ policy on declaration of interests and have no relevant interests to declare.

Provenance and peer review: Not commissioned; externally peer reviewed.

1 Cancer Research UK. Cervical cancer incidence statistics. 2014. www.cancerresearchuk. org/cancer-info/cancerstats/types/cervix/incidence/\#age.

2 Cancer Research UK. Cervical cancer mortality statistics. 2014. www.cancerresearchuk. org/cancer-info/cancerstats/types/cervix/mortality.

3 Castanon A, Landy R, Cuzick J, Sasieni P. Cervical screening at age 50-64 years and the risk of cervical cancer at age 65 years and older: population-based case control study. PLoS Med 2014;11:e1001585.

4 NHS Cervical Screening Programme. Statistical bulletin (England 2012-13). www. cancerscreening.nhs.uk/cervical/statistics-bulletin.html.

5 Waller J, McCaffery K, Wardle J. Beliefs about the risk factors for cervical cancer in a British population sample. Prev Med 2004;38:745-53.

6 Cancer Research UK. Breast cancer screening statistics. 2012. www.cancerresearchuk. org/cancer-info/cancerstats/types/breast/screening/Programme-Activity/\#age.
7 Lancucki L, Sasieni P, Patnick J, Day TJ, Vessey MP. The impact of Jade Goody's diagnosis and death on the NHS cervical screening programme. J Med Screen 2012;19:89-93.

8 Marlow LA, Sangha A, Patnick J, Waller J. The Jade Goody effect: whose cervical screening decisions were influenced by her story? J Med Screen 2012;19:184-8.

9 Low EL, Simon AE, Lyons J, Romney-Alexander D, Waller J. What do British women know about cervical cancer symptoms and risk factors? Eur J Cancer 2012:48:3001-8.

10 Stevens JC, Cain WS. Old-age deficits in the sense of smell as gauged by thresholds, magnitude matching, and odor identification. Psychol Aging 1987;2:36.

11 Sasieni P, Adams J, Cuzick J. Benefit of cervical screening at different ages: evidence from the UK audit of screening histories. Br J Cancer 2003;89:88-93.

12 NHS Cervical Screening Programme. Audit of invasive cervical cancer. 2012. www. cancerscreening.nhs.uk/cervical/publications/nhscsp-audit-invasive-cervical-cancer201205.pdf.

13 Office of National Statistics. Interim life tables 2014. www.ons.gov.uk/ons/taxonomy/index. html?nscl=Interim+Life+Tables.

14 Elit L. Role of cervical screening in older women. Maturitas 2014;79:413-20.

15 Moser K, Patnick J, Beral V. Inequalities in reported use of breast and cervical screening in Great Britain: analysis of cross sectional survey data. BMJ 2009;338:b2025.

16 Von Wagner C, Macedo A, Campbell C, et al. Continuing cancer screening later in life: attitudes and intentions among older adults in England. Age Ageing 2013;42:770-5.

17 Karwalajtys T, Howard M, Sellors JW, Kaczorowski J. Vaginal self sampling versus physician cervical sampling for HPV among younger and older women. Sex Transm Infect 2006;82:337-9.

18 Haguenoer K, Sengchanh S, Gaudy-Graffin C, et al. Vaginal self-sampling is a cost-effective way to increase participation in a cervical cancer screening programme: a randomised trial. Br J Cancer 2014;111:2187-96.

Accepted: 21 April 2015

Cite this as: BMJ 2015;350:h2729

(c) BMJ Publishing Group Ltd 2015 


\section{Table}

Table 1/ New cases (2009-11) of cervical cancer and deaths (2010-12) in UK by age

\begin{tabular}{cccccc}
\multirow{2}{*}{ Age } & \multicolumn{2}{c}{ New cases } & & \multicolumn{2}{c}{ Deaths } \\
\cline { 2 - 3 } \cline { 5 - 6 } & No & No/100 000* & & No & No/100 000* \\
\hline 15 & 0 & 0 & & 0 \\
\hline $15-19$ & 2 & 0.1 & & 0 \\
\hline $20-24$ & 62 & 2.9 & & 0.3 \\
\hline $25-29$ & 410 & 19.1 & 27 & 1.3 \\
\hline $30-34$ & 398 & 19.7 & 36 & 1.7 \\
\hline $35-39$ & 411 & 19.1 & 52 & 2.5 \\
\hline $40-44$ & 369 & 15.7 & 67 & 2.9 \\
\hline $45-49$ & 280 & 12.1 & 84 & 3.6 \\
\hline $50-54$ & 209 & 10.3 & 74 & 3.6 \\
\hline $55-59$ & 189 & 10.4 & 68 & 3.7 \\
\hline $60-64$ & 175 & 9.1 & 79 & 4.1 \\
\hline $65-69$ & 131 & 8.6 & 80 & 5.0 \\
\hline $70-74$ & 128 & 9.9 & 84 & 6.5 \\
\hline $75-79$ & 131 & 11.8 & 90 & 8.1 \\
\hline $80-84$ & 110 & 12.5 & 82 & 9.3 \\
\hline$\geq 85$ & 116 & 12.4 & 113 & 11.9 \\
\hline
\end{tabular}

*Per 100000 women in each age group. 